\title{
Thoracic Paravertebral Nerve Block with Ropivacaine and Adjuvant Dexmedetomidine Produced Longer Analgesia in Patients Undergoing Video-Assisted Thoracoscopic Lobectomy: A Randomized Trial
}

\author{
Jun Zha $\mathbb{D}^{1}$, Shiliang Ji $\mathbb{D}^{2}{ }^{2}$ Chen Wang $\mathbb{D}^{1},{ }^{1}$ Zhe Yang $\mathbb{D}^{1},{ }^{1}$ Shigang Qiao $\mathbb{D}^{1,3}$ \\ and Jianzhong An $\mathbb{D}^{3}$ \\ ${ }^{1}$ Department of Anesthesiology and Perioperative Medicine, \\ The Affiliated Suzhou Science \& Technology Town Hospital of Nanjing Medical University, No. 1 Lijiang Road, \\ Suzhou 215153, China \\ ${ }^{2}$ Department of Pharmacy, The Affiliated Suzhou Science \& Technology Town Hospital of Nanjing Medical University, \\ No. 1 Lijiang Road, Suzhou 215153, China \\ ${ }^{3}$ Institute of Clinical Medicine Research, \\ The Affiliated Suzhou Science \& Technology Town Hospital of Nanjing Medical University, No. 1 Lijiang Road, \\ Suzhou 215153, China
}

Correspondence should be addressed to Shigang Qiao; qiaoshigang@163.com and Jianzhong An; szkjcyy_ajz@126.com

Received 23 July 2021; Revised 11 August 2021; Accepted 21 August 2021; Published 8 September 2021

Academic Editor: Enas Abdulhay

Copyright ( 12021 Jun Zha et al. This is an open access article distributed under the Creative Commons Attribution License, which permits unrestricted use, distribution, and reproduction in any medium, provided the original work is properly cited.

\begin{abstract}
Purpose. This study evaluated the postoperative analgesic effect of ultrasound-guided single-point thoracic paravertebral nerve block (TPVB) combined with dexmedetomidine (DEX) in patients undergoing video-assisted thoracoscopic lobectomy. Methods. Sixty adult patients of the American Society of Anesthesiologists (ASA) I-III were randomly assigned into three groups ( $n=20$ each). G group: patients received routine general anesthesia; PR group: patients received 0.5\% ropivacaine; and PRD group: patients received $0.5 \%$ ropivacaine with $1 \mu \mathrm{g} / \mathrm{kg}$ DEX. TPVB was performed in the T5 space before surgery, and then, general anesthesia induction and video-assisted thoracoscopic lobectomy were performed. Analgesics were administered through the patient-controlled analgesia (PCA) device intravenously. The background infusion of each PCA device was set to administer $0.02 \mu \mathrm{g} / \mathrm{kg} / \mathrm{h}$ sufentanil, with a lockout time of $15 \mathrm{~min}$, and a total allowable volume is $100 \mathrm{ml}$. Results. Compared to PR and G groups, the total sufentanil consumption after operation, the times of analgesic pump pressing, the pain score, and the incidence of postoperative nausea or vomiting in the PRD group were significantly reduced $(p<0.05)$. Also, the duration of first time of usage of the patient-controlled analgesia (PCA) was longer. The heart rate (HR) and mean arterial pressure (MAP) during operation were lower in the PRD group as compared with the other two groups in most of the time. However, hypotension and arrhythmia occurred in three groups with no statistically significant difference. Conclusions. A small volume of TPVB with ropivacaine and DEX by single injection produced longer analgesia in patients undergoing video-assisted thoracoscopic lobectomy, reduced postoperative opioids consumption, and the incidence of side effects.
\end{abstract}

\section{Introduction}

Video-assisted thoracoscopic surgery (VATS) is mainly used for the treatment of the lung, mediastinum, and pleural lesions. The main advantage is to avoid the injury of thoracotomy. Compared with thoracotomy, the operation time is shorter, the postoperative morbidity is lower, and the time for returning to normal activities is earlier [1]. However, effective analgesia is still needed to reduce postoperative pain and postoperative nausea and vomiting. 
Thoracic paravertebral block (TPVB) as part of a multimodal analgesia strategy after thoracotomy [2] and breast surgery [3] has a broad evidence base and along with ultrasound-guided techniques. It has become increasingly popular. Recent randomized controlled trials and reviews have shown that paravertebral block (PVB) causes prolonged directional analgesia and reduces the risk of postoperative nausea, vomiting, and complications $[4,5]$. However, a large body of literature has shown that the duration of analgesia is controversial, and PVB is only beneficial immediately after surgery $[6,7]$. There are few studies on the effectiveness and tolerability of adjuvant analgesics in paraspinal analgesia. The addition of magnesium, clonidine, ketamine, dexamethasone, opioids, and other analgesics and local anesthetics can enhance and prolong the analgesic effect provided by $\operatorname{PVB}[8,9]$.

Dexmedetomidine (DEX) is a novel alpha-2 adrenergic receptor agonist with dose-dependent sedative, anxiolytic, and analgesic effects and has an advantage of minimum respiratory depression compared with alternative drugs [10]. It is well known that $\alpha_{-2}$ receptor agonists, due to their own sympathetic properties, provide stable hemodynamics during surgery and reduce the amount of narcotic analgesics. When DEX is used as an anesthetic for epidural anesthesia [11], subarachnoid block $[12,13]$, PVB $[14,15]$, and brachial plexus block analgesia [16], the time is significantly extended. However, there are few studies evaluating the efficacy of DEX as an adjuvant in the treatment of analgesia after thoracoscopic surgery, only in partial breast surgery [15].

Therefore, this study was designed to evaluate whether TPVB combined with ropivacaine and DEX could improve the analgesic effect of patients undergoing VATS, thereby reducing postoperative opioid drugs consumption.

\section{Methods}

This study was approved by the Affiliated Suzhou Science and Technology Town Hospital of Nanjing Medical University' Ethics Committee, and written informed consent was obtained from all patients. The trial is registered at the Chinese Clinical Trial Registry, number ChiCTR-IOR17013034. After obtaining approval and written informed consent from the institutional ethics committee, the study enrolled 60 ASA I-III patients (18-65 years old, either gender, weighing 50-85 kg) who were scheduled to undergo elective thoracoscopic lobectomy. Patients undergoing elective general anesthesia and video-assisted thoracoscopic unilateral lobectomy had no history of cardiopulmonary disease, no sinus bradycardia, no functional lesions of the sinoatrial node, no serious arrhythmia, and no thoracic deformity. Surgery time was more than 2 hours. Exclusion criteria: minimental state examination scores below 23 points; the history of dementia, psychosis, or other central nervous system disease or drug dependence and poor compliance; and those who did not complete video-assisted thoracoscopic lobectomy under general anesthesia or have to convert to thoracotomy.

All patients did not use preoperative medication, and in the preoperative preparation room, the patients were explained in detail the numerical rating scale (NRS) scoring rules (scores from 0 to 10: $0=$ no pain, $10=$ most severe pain). Patients were randomized into three groups of 20 individuals each using a computer-generated random number table (random number table method) with sealed envelope technology for assignment concealment (distribution hidden). In the operating room, ultrasound-guided right internal jugular vein catheterization for infusion and left radial artery catheterization for monitoring the changes of pressure have been performed. Conventional general anesthesia induction included sufentanil $5 \mathrm{ug} /$ $\mathrm{kg}$ + etomidate $2 \mathrm{mg} / \mathrm{kg}$ + cisatracurium $2 \mathrm{mg} / \mathrm{kg}$ intravenous slow bolus injection; anesthesia maintenance: propofol $2.5 \mathrm{ug} / \mathrm{ml}$, sevoflurane $0.8 \mathrm{MAC}$, remifentanil $3 \mathrm{ng} / \mathrm{ml}$, bispectral index monitoring, and the depth of anesthesia is maintained between 40 and 60. Patients were classified into three groups as follows: G group: patients received routine general anesthesia; PR group: patients received $0.5 \%$ ropivacaine for TPVB; PRD group: patients received $0.5 \%$ ropivacaine with $1 \mu \mathrm{g} / \mathrm{kg}$ DEX for TPVB. Paravertebral blockade was performed under ultrasound guidance. A linear ultrasound transducer (HITACHI Arietta 60) was placed intercostally to identify the thoracic paravertebral space (TPVS), and a 20-gauge needle was inserted into the plane of the transducer. When the needle tip reached TPVS, $10 \mathrm{~mL} 0.5 \%$ ropivacaine with $1 \mu \mathrm{g} / \mathrm{kg}$ DEX in T5 paravertebral space was injected.

The gender, age, ASA grade, height, weight, body mass index (BMI), heart rate (HR), mean artery pressure (MAP) baseline, duration of surgery (h), anesthesia (h), the time to first analgesic request since paravertebral injection (TFR1), first use of patient-controlled analgesia (PCA1) intravenously, total sufentanil dosage, and the pressing times of analgesic pumps were all recorded in three groups. The intraoperative MAP and HR were measured after starting TPVB bolus injection. Hypotension defined as a $30 \%$ decrease or less than $80 \mathrm{mmHg}$ in systolic blood pressure from baseline was treated with ephedrine $5 \mathrm{mg}$ intravenously and further boluses as required. Bradycardia defined as heart rate $<55$ beats per minute was treated with $0.6 \mathrm{mg}$ intravenously. Analgesics were administered through the PCA device (ZZB-150, Apon, Nantong, China). The background infusion of each PCA device was set to administer $0.02 \mu \mathrm{g} / \mathrm{kg} / \mathrm{h}$ sufentanil, with a lockout time of $15 \mathrm{~min}$, and a total allowable volume is $100 \mathrm{ml}$. The anesthetist conducted postoperative monitoring, pain assessment, and management was blinded to the patient groups. The flowchart of the study protocol is shown in Figure 1.

Ramsay sedation scores of patients were recorded at 30 minutes, 1 hour, 2 hours, 4 hour, 6 hours, 8 hours, 12 hours, and 24 hours after anesthetic recovery in three groups (score 1: patient anxiety, agitated or restlessness, or both; score 2: patient cooperation, orientation, and calm; score 3: patient only responds to orders; score 4: patient with light outer membrane percussion, or a loud auditory stimuli showed a rapid response; score 5: the patient showed a slow response to light eyebrows or loud auditory stimuli; score 6: the patient did not respond; score $2-4$ is an ideal sedation level). The intraoperative MAP and $\mathrm{HR}$ were recorded at 10 


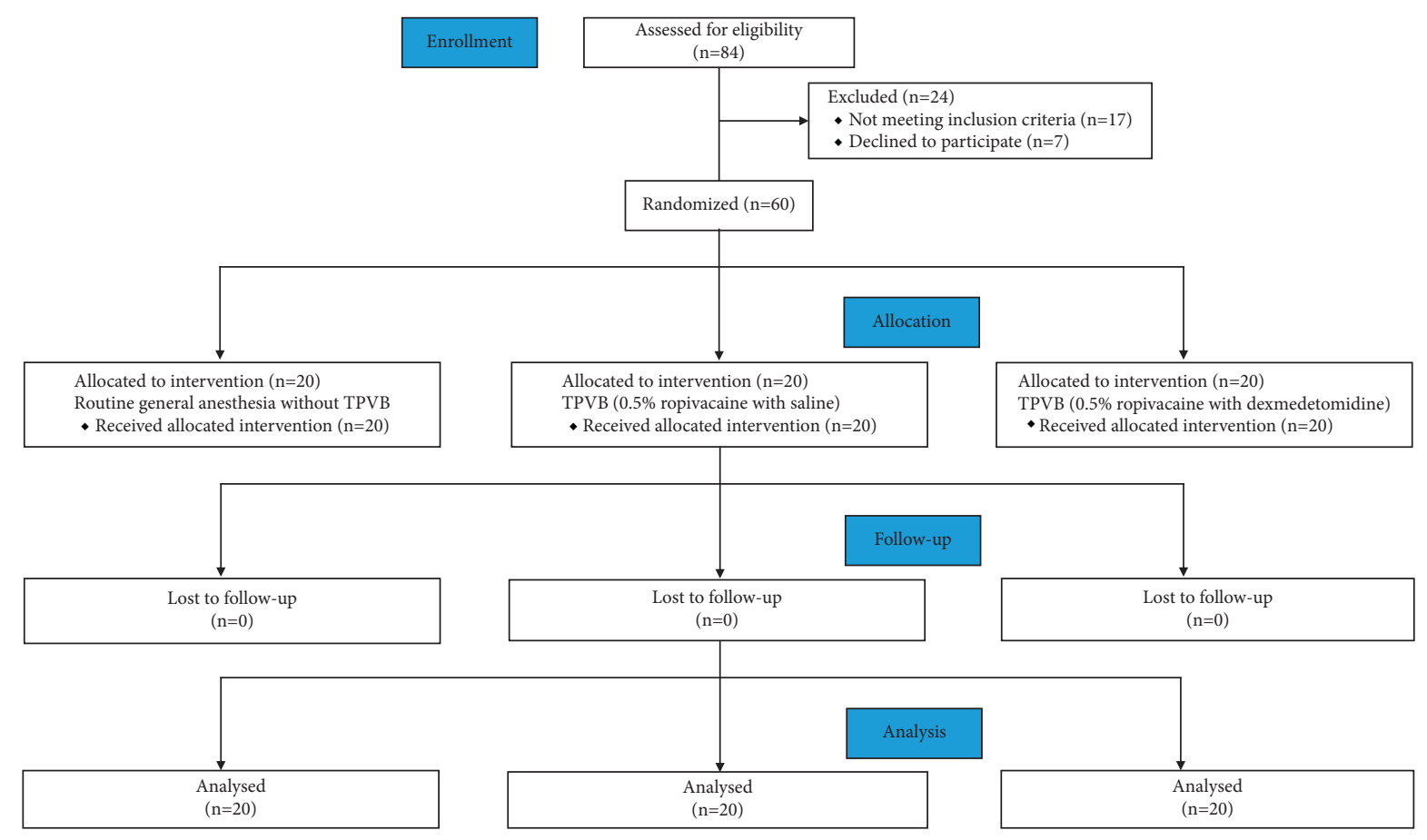

FIgURE 1: CONSORT flow diagram of the study.

minutes, $20 \mathrm{~min}, 30 \mathrm{~min}, 40 \mathrm{~min}, 50 \mathrm{~min}, 60 \mathrm{~min}, 70 \mathrm{~min}$, $80 \mathrm{~min}$, $90 \mathrm{~min}$, and $100 \mathrm{~min}$ after starting TPVB bolus injection. Postoperative pain ratings (NRS) during rest and movement were recorded every two hours within 24 hours (NRS: NRS uses 0-10 to represent different degrees of pain. Score 0: no pain; score 1-3: mild pain; score 4-6: moderate pain; score 7-10: severe pain).

All values are mean \pm SEM. Two-way ANOVA for repeated measures was used when appropriate (SPSS 20.0 for Windows, SPSS Inc.). The Student-Newman-Keuls multiple comparison post hoc test was used to differentiate within the groups. A probability value less than 0.05 was considered to indicate a significant difference between the groups, while a value greater than 0.05 was considered to indicate no significant difference between the groups.

\section{Results}

There were no significant differences among the G group, $\mathrm{PR}$ group, and PRD group in demographic data such as age, weight, height, BMI, and baseline hemodynamic parameters durations of surgery and anesthesia $(p>0.05)$ (Table 1). All patients underwent the surgery successfully without local anesthetic toxicity or diclofenac sodium contraindications.

Postoperative sufentanil (over 24 hours) consumptions were reduced significantly in the PRD group compared to other two groups $(p<0.05$; Table 2$)$. Moreover, all the abovementioned parameters did not reach significant difference between the $G$ group and PR group as given in Table $2(p>0.05)$. There was no significant difference of postoperative Ramsay sedation scores among all groups before $12 \mathrm{~h}$; however, the score values in the G group were significantly lower than that in the $\mathrm{PR}$ or $\mathrm{PRD}$ group $(p<0.05$; Table 3$)$.

The NRS pain scores of ipsilateral arm were, respectively, indicated in Figure 2 and 3 in rest or movement status. There was no significant difference in pain score among three groups in rest or movement status within 1 hour after surgery; 2 hours after surgery, the pain scores of the PRD group were lower than other two groups. In the PRD group, TFR1 and PCA1 were significantly longer than those in the PR group $(p<0.05)$ and $G$ group $(p<0.05)$ (Table 2$)$. There were significant differences of TFR1 and PCA1 between the PR group and $G$ group $(p<0.05)$.

Hemodynamic parameters were monitored during surgery. HR and MAP trends are shown in Figures 4 and 5, respectively. There were no differences of hypotension and bradycardia among groups and the requirement of vasopressors for maintenance of stable hemodynamic parameters during the induction period did not show significant differences among groups (data not shown). The intraoperative $\mathrm{HR}$ and MAP in the PRD group were lower compared to other two groups. After 10 minutes, the intraoperative HR of all three groups decreased, but it was more obvious in the PR group and G group than that in the PRD group $(p<0.05)$. In the PR group and $G$ group, the intraoperative HR tended to stabilize and increased slowly until 40 minutes, but the HR of the PRD group was lower than other $\mathrm{G}$ groups at 80 and 100 minutes. In the PR group and G group, the intraoperative MAP decreased significantly around 20 minutes, while MAP of the PRD group decreased to $(77.1 \pm 1.7) \mathrm{mmHg}$ around 40 minutes, and it gradually returned to the baseline level around 100 minutes $(p<0.05)$. However, there was no significant difference in the consumption of sufentanil dose or the 
TABLE 1: Characteristics of the patients and surgery.

\begin{tabular}{|c|c|c|c|c|}
\hline Items & G group $(n=20)$ & PR group $(n=20)$ & PRD group $(n=20)$ & $P$ value \\
\hline Age (years) & $45.7 \pm 1.3$ & $47.1 \pm 1.7$ & $48.1 \pm 1.5$ & 0.962 \\
\hline Height $(\mathrm{cm})$ & $165.4 \pm 1.4$ & $165.9 \pm 1.2$ & $164.6 \pm 1.4$ & 0.594 \\
\hline Weight (kg) & $68.0 \pm 2.1$ & $67.3 \pm 2.3$ & $72.4 \pm 2.1$ & 0.719 \\
\hline $\operatorname{BMI}\left(\mathrm{kg} / \mathrm{m}^{2}\right)$ & $23.2 \pm 0.5$ & $23.4 \pm 0.6$ & $24.1 \pm 0.6$ & 0.574 \\
\hline Baseline HR (beats/min) & $83.0 \pm 1.6$ & $82.3 \pm 2.9$ & $84.8 \pm 2.3$ & 0.922 \\
\hline Baseline MAP (mmHg) & $96.7 \pm 1.8$ & $97.1 \pm 1.5$ & $95.5 \pm 1.9$ & 0.460 \\
\hline Surgery time $(\mathrm{h})$ & $2.2 \pm 0.1$ & $2.2 \pm 0.1$ & $2.2 \pm 0.1$ & 0.614 \\
\hline Anesthesia time (h) & $2.5 \pm 0.1$ & $2.8 \pm 0.1$ & $2.9 \pm 0.1$ & 0.067 \\
\hline
\end{tabular}

Values are mean \pm SEM. G: general anesthesia; PR, paravertebral ropivacaine; PRD, paravertebral ropivacaine and dexmedetomidine; BMI, body mass index; $\mathrm{HR}$, heart rate; MAP, mean arterial pressure.

TABLe 2: Analgesic efficacy and postoperative adverse effects.

\begin{tabular}{|c|c|c|c|c|}
\hline Items & G group $(n=20)$ & PR group $(n=20)$ & PRD group $(n=20)$ & $P$ value \\
\hline TFR1 (h) & $2.6 \pm 1.5$ & $5.6 \pm 2.0$ & $24.1 \pm 2.9$ & $<0.05^{* \# \$}$ \\
\hline Numbers of pressing analgesic pump & $5.3 \pm 0.2$ & $3.2 \pm 0.1$ & $1.6 \pm 0.2$ & $<0.05^{* \# \$}$ \\
\hline Total sufentanil dosage $(\mu \mathrm{g})$ & $142.2 \pm 7.3$ & $105.1 \pm 5.7$ & $78.2 \pm 6.2$ & $<0.05^{*} \# \$$ \\
\hline Nausea & $5(25 \%)$ & $3(15 \%)$ & 0 & $<0.05^{* \# \$}$ \\
\hline Vomiting & $2(10 \%)$ & $1(5 \%)$ & 0 & $<0.05^{* \# \$}$ \\
\hline
\end{tabular}

Values are mean \pm SEM or $n$ (\%). TFR1, time from paravertebral injection to first analgesic request. $P<0.05,{ }^{*} \mathrm{G}$ group vs. PR group; ${ }^{\#} \mathrm{G}$ group vs. $\mathrm{PRD}$ group; ${ }^{\$}$ PR group vs. PRD group.

TABLE 3: Postoperative Ramsay sedation scores at different intervals.

\begin{tabular}{|c|c|c|c|c|}
\hline Time points & G group $(n=20)$ & PR group $(n=20)$ & PRD group $(n=20)$ & $P$ value \\
\hline $30 \mathrm{~min}$ & $2.5(2.0-5.0)$ & $2.5(2.0-5.0)$ & $2.0(2.0-5.0)$ & 0.409 \\
\hline $1 \mathrm{~h}$ & $2.0(2.0-4.0)$ & $2.0(2.0-4.0)$ & $2.0(2.0-4.0)$ & 0.995 \\
\hline $2 \mathrm{~h}$ & $2.0(2.0-4.0)$ & $2.0(2.0-4.0)$ & $2.0(2.0-4.0)$ & 1.000 \\
\hline $4 \mathrm{~h}$ & $2.0(2.0-3.0)$ & $2.0(2.0-3.0)$ & $2.0(2.0-3.0)$ & 0.415 \\
\hline $8 \mathrm{~h}$ & $2.0(2.0-3.0)$ & $2.0(2.0-3.0)$ & $2.0(2.0-3.0)$ & 0.216 \\
\hline $12 \mathrm{~h}$ & $2.0(2.0-3.0)$ & $2.0(2.0-3.0)$ & $2.0(2.0-3.0)$ & 0.331 \\
\hline $24 \mathrm{~h}$ & $1.0(0.0-2.0)$ & $2.0(1.0-3.0)$ & $2.0(0.0-3.0)$ & $<0.05^{* \#}$ \\
\hline
\end{tabular}

Values as median (interquartile range). ${ }^{*} \mathrm{G}$ group versus PR group; ${ }^{*} \mathrm{G}$ group versus $\mathrm{PRD}$ group.

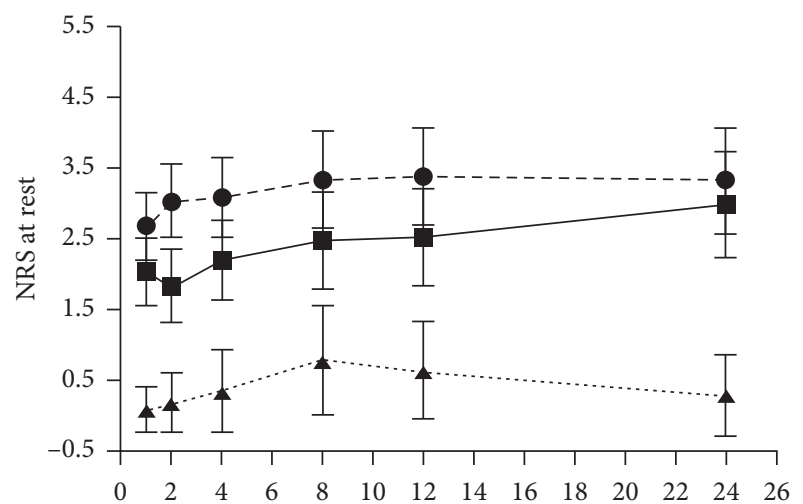

(h)

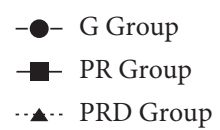

FIgUre 2: Postoperative NRS.R. Values in all groups. Values in the PRD group compared to the G group and the PR group at all time points. There was no difference between the $G$ group and $P R$ group $(p>0.05)$.

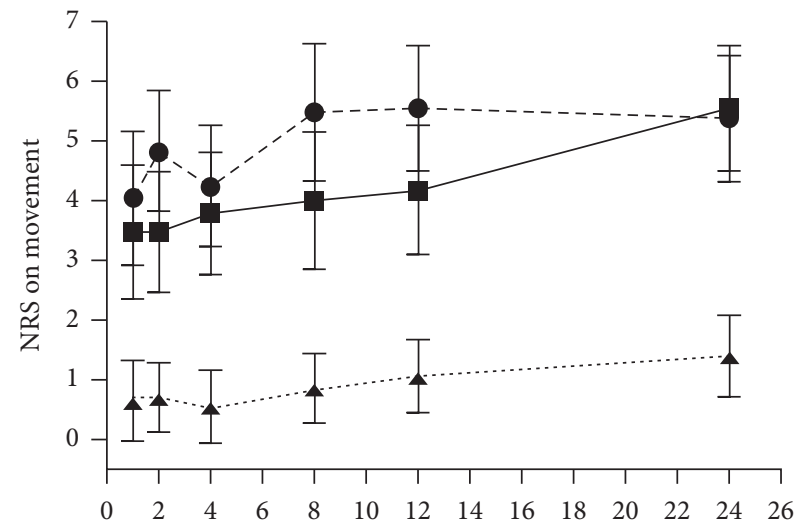

(h)

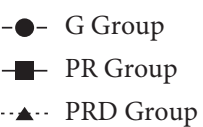

FIgURe 3: Postoperative NRS.M. Values in all groups. Values in the PRD group compared to the $G$ group and the PR group at all time points $(p<0.05)$. There was no difference between the $\mathrm{G}$ group and PR group. 


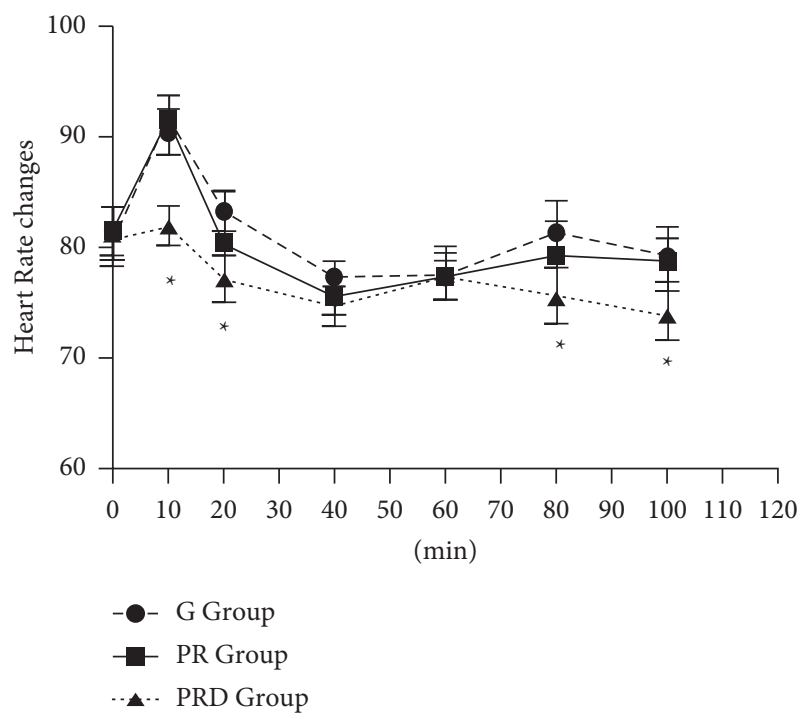

FIgURE 4: Intraoperative heart rate values in all groups. The PRD group compared to the $\mathrm{G}$ group and $\mathrm{PR}$ group except at 40 minutes and 60 minutes $\left({ }^{*} p<0.05\right)$.

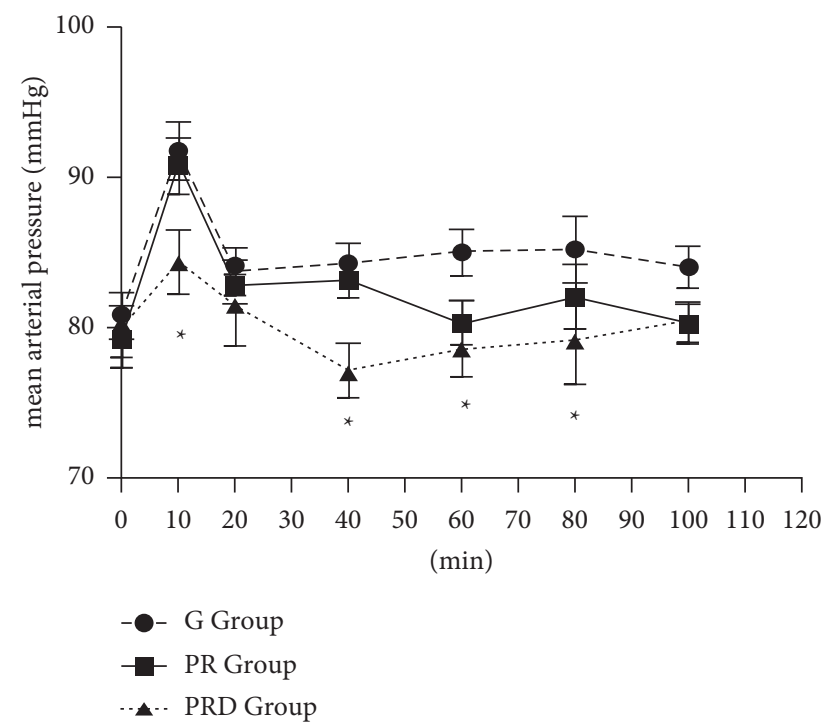

FIGURE 5: Intraoperative MAP values in all groups. The PRD group compared to the $\mathrm{G}$ group and $\mathrm{PR}$ group except at $20 \mathrm{~min}$ and $100 \mathrm{~min}\left({ }^{*} p<0.05\right)$. There were significant differences between the G group and PR group at 60, 80, and then 100 min; the G group and PRD group at all time points except $20 \mathrm{~min}$; and the PR group and PRD group at 20 and $100 \mathrm{~min}(p<0.05)$.

incidence of hypotension and the occurrence of bradycardia during the surgery.

\section{Discussion}

TPVB has been used wildly to reduce postoperative opioid consumption and provide effective pain control. The injection site and volume are quite different in different studies, and there are single and multiple injections with volumes of $5,7,10,15,20$, or $0.3 \mathrm{ml} / \mathrm{kg}$ [17]. A cadaver study observed distribution of $20 \mathrm{ml}$ injected dye over three to four
TPVS (range, 1-10) with 40\% incidence of epidural spread [2]. Therefore, the analgesic effect produced by the volume of 15 or $20 \mathrm{ml}$ may also include the effect of the epidural spread. In this study, we applied a volume of $10 \mathrm{ml}$ of DEX combined with ropivacaine TPVB for patients with thoracoscopic lobectomy had a better analgesic effect than patients with only TPVB or no TPVB and may reduce the incidence of epidural spread.

DEX acts as a potential adjuvant for both axons and peripheral nerve blocks $[16,18]$, and studies have confirmed that epidural injection of DEX enhanced local anesthetics on nerves, reduced the need for intraoperative anesthesia, and provided a better analgesic effect after thoracotomy [19]. The analgesic effect is concentrated by inhibiting the release of substance $\mathrm{P}$ in the nociceptive pathway of dorsal root neurons and activating the alpha-2 receptor in the blue spot. This alpha- 2 agonist mediates peripheral analgesia by reducing the release of norepinephrine and the independent inhibition of neurofibrillary action potential by the alpha- 2 receptor. None of the various animal studies showed any adverse neurological effects of DEX $[20,21]$ and neuroprotective effects of dexmedetomidine, induced by intrathecal administration is similar to methylprednisolone [21]. DEX has also been used in human studies as an adjuvant for local anesthetics $[14,15]$. Mahendru et al. compared the effects of intrathecal DEX and fentanil as bupivacaine adjuvants; the results showed that intrathecal DEX prolonged exercise and sensory block, more hemodynamic stability, and reduced need for analgesics within 24 hours compared with fentanil [22]. In other studies, DEX improved blocker efficiencies have been shown, with no reported neurological side effects. Further study of the neuronal effects of DEX was encouraged by the researchers $[15,16]$. In the current study, the consumption of sufentanil in the two paravertebral nerve block patients was significantly lower than that in the control group, indicating that paravertebral nerve block can prolong the analgesic time. None of the patients in the PRD group required an additional dose of sufentanil, while only one patient in the PR group required an additional dose of sufentanil. Compared with the PR group and the G group, the first analgesia request time was much longer in the PRD group, and the postoperative PRD group had a longer PCA1 time, but the total number of PCA (or sufentanil consumption) and pain score are lower. This indicates that ropivacaine supplemented with DEX prolongs the time of analgesia after PVB. In addition, patients receiving DEX had less opioid-related complications such as nausea and vomiting. On the other hand, all of these parameters were comparable in the PR group and G group, indicating that there was no significant TPVB analgesic effect of ropivacaine alone compared with patients who did not receive TPVB. The use of local anesthetic TPVB alone improved intraoperative analgesia and reduced the need for sufentanil, but did not provide adequate postoperative analgesia. A recent RCT meta-analysis study evaluated the analgesic effects of multilevel TPVB in combination with ropivacaine or bupivacaine in postoperative breast cancer [23], and the results showed the overall analgesic consumption was lower in the intraoperative and postoperative ropivacaine groups 
or bupivacaine group, and the number of patients with NRS $>3$ after PACU was significantly reduced in the TPVB group. The local anesthetic infiltration group immediately reduced postoperative pain, especially in the 2 hours after surgery, but there was no significant difference of analgesia between 12 hours and 24 hours after surgery.

In this study, the requirement of vasopressors for maintenance of stable hemodynamic parameters did not reveal any significant difference during the induction period among groups. However, the HR and MAP in the PRD group were lower than those in the other two groups during surgery. It has been reported that the stable hemodynamics may possibly be explained on the basis of lower volume of local anesthetics used and a suitable selection of the dose of adjuvant [11]. The sedative effects found in the study may be related to the use of DEX [24]. A higher sedation score in the $\mathrm{PR}$ and PRD groups than the G group may due to better analgesia effects in PR and PRD groups.

More recently, Hong et al. reported that DEX as an adjunct in TPVB provided effective pain relief and significantly reduced opioid requirement in VATS [25]. Our results are consistent with the Hong et al.' study. However, there are two differences between our study and Hong et al. study'. (1) In our study, only on injection at T5 space before surgery, while there are two injections at the T3-T4 and T4T5 levels after surgery in Hong et al.' study. It has been reported that TPVB performed prior to general anesthesia for laparoscopic cholecystectomy can provide early discharge and better postoperative pain management [6]. (2) The injection volume of the ropivacaine and DEX is different. The injection volume of ropivacaine and DEX mixture in our study is $10 \mathrm{ml}$ at the single site, while the total volume of the two site injections was $30 \mathrm{ml}$ in Hong et al.' study. Our results demonstrated that a small injection volume ( $10 \mathrm{ml} 0.5 \%$ ropivacaine with $1 \mu \mathrm{g} / \mathrm{kg}$ DEX) at site of $\mathrm{T} 5$ space before surgery also achieved effective postoperative analgesia. It may have beneficial clinical effects for reducing the side effects of drugs, puncture injury, and discomfort to patients and enhancing recovery after surgery.

There are certain limitations to our study. First, it was a retrospective study with a small sample size and the findings therefore have to be confirmed with a larger randomized controlled trial. Second, the small sample size and recruitment of relatively healthy patients (ASA I and II) limited the possibility of drawing a definitive conclusion. Greater numbers of patients and patients with multiple comorbidities (i.e., ASA III or IV) need to be included in future studies to verify these findings. Third, since a single dose of TPVB can provide sufficient pain relief, inserting a catheter into the paravertebral space for continuous infusion of local anesthetics may provide protracted postsurgery analgesia. Furthermore, different doses and drugs used in TPVB may cause bias of the results. More studies have to be conducted to clarify this question.

\section{Conclusions}

In summary, a small volume of TPVB with $1 \mu \mathrm{g} / \mathrm{kg}$ DEX combined with $0.5 \%$ ropivacaine by single injection at $\mathrm{T} 5$ space before surgery for patients undergoing thoracoscopic lobectomy prolonged the overall analgesia time after surgery and reduced the dose and side effects of opioids.

\section{Data Availability}

The data used to support the findings of this study are available from the corresponding author upon request.

\section{Disclosure}

The funding body had no role in the design of this study, analyses, interpretation of the data, or writing the manuscript.

\section{Conflicts of Interest}

The authors declare that they have no conflicts of interest.

\section{Authors' Contributions}

Jun Zha and Shiliang Ji contributed equally to this work.

\section{Acknowledgments}

This work was supported by grants QNRC2016219 from Jiangsu Key Talent Youth Awards in Medicine, GSWS2019092 from Gusu Health Youth Talent Awards, and $2016 Z 002$ (to Dr. Qiao) and SGXWS2020 (to Dr. Ji) from the Third Batch of Suzhou High-Tech District Health Talents Project. The funding body contributed with research equipment.

\section{References}

[1] S. Kaseda, T. Aoki, N. Hangai, and K. Shimizu, "Better pulmonary function and prognosis with video-assisted thoracic surgery than with thoracotomy," The Annals of Thoracic Surgery, vol. 70, no. 5, pp. 1644-1646, 2000.

[2] A. C. Krediet, N. Moayeri, G. J. V. Geffen et al., "Different approaches to ultrasound-guided thoracic paravertebral block," Anesthesiology, vol. 123, no. 2, pp. 459-474, 2015.

[3] A. Schnabel, S. U. Reichl, P. Kranke, E. M. Z. Pogatzki, and P. K. Zahn, "Efficacy and safety of paravertebral blocks in breast surgery: a meta-analysis of randomized controlled trials," British Journal of Anaesthesia, vol. 105, no. 6, pp. 842-852, 2010.

[4] Y. Tahiri, D. Q. H. Tran, J. Bouteaud et al., "General anaesthesia versus thoracic paravertebral block for breast surgery: a meta-analysis," Journal of Plastic, Reconstructive \& Aesthetic Surgery, vol. 64, no. 10, pp. 1261-1269, 2011.

[5] J. F. Moller, L. Nikolajsen, S. A. Rodt, H. Ronning, and P. S. Carlsson, "Thoracic paravertebral block for breast cancer surgery: a randomized double-blind study," Anesthesia and Analgesia, vol. 105, no. 6, pp. 1848-1851, 2007.

[6] Z. M. Naja, M. E. Rajab, F. Ziade, M. A. Tannir, and T. Itani, "Preoperative vs. postoperative bilateral paravertebral blocks for laparoscopic cholecystectomy: a prospective randomized clinical trial," Pain Practice, vol. 11, no. 6, pp. 509-515, 2011.

[7] J. C. Boughey, F. Goravanchi, R. N. Parris, S. S. Kee, A. M. Kowalski, and J. C. Frenzel, "Prospective randomized trial of paravertebral block for patients undergoing breast 
cancer surgery," The American Journal of Surgery, vol. 198, no. 5, pp. 720-725, 2009.

[8] S. S. Liu and F. V. Salinas, "Continuous plexus and peripheral nerve blocks for postoperative analgesia," Anesthesia and Analgesia, vol. 96, no. 1, pp. 263-272, 2003.

[9] M. E. Mourad and A. Amer, "Effects of adding dexamethasone or ketamine to bupivacaine for ultrasound-guided thoracic paravertebral block in patients undergoing modified radical mastectomy: a prospective randomized controlled study," Indian Journal of Anaesthesia, vol. 62, no. 4, pp. 285-291, 2018.

[10] L. Wang, Y. Zhou, T. Zhang, L. Huang, and W. Peng, "Comparison in sedative effects between dexmedetomidine and midazolam in dental implantation: a randomized clinical trial," BioMed Research International, vol. 2020, Article ID 6130162, 7 pages, 2020.

[11] S. J. S. Bajwa, A. Singh, V. Arora, J. Kaur, and S. Parmar, "Comparative evaluation of dexmedetomidine and fentanyl for epidural analgesia in lower limb orthopedic surgeries," Saudi Journal of Anaesthesia, vol. 5, no. 4, pp. 365-370, 2011.

[12] R. Gupta, J. Bogra, R. Verma, M. Kohli, J. K. Kushwaha, and S. Kumar, "Dexmedetomidine as an intrathecal adjuvant for postoperative analgesia," Indian Journal of Anaesthesia, vol. 55, pp. 347-351, 2011.

[13] R. Gupta, R. Verma, J. Bogra, M. Kohli, R. Raman, and J. K. Kushwaha, "A comparative study of intrathecal dexmedetomidine and fentanyl as adjuvants to Bupivacaine," Journal of Anaesthesiology, Clinical Pharmacology, vol. 27, pp. 339-343, 2011.

[14] S. A. Mohamed, K. M. Fares, A. A. Mohamed, and N. H. Alieldin, "Dexmedetomidine as an adjunctive analgesic with bupivacaine in paravertebral analgesia for breast cancer surgery," Pain Physician, vol. 17, no. 5, pp. E589-E598, 2014.

[15] M. Mohta, B. Kalra, A. K. Sethi, and N. Kaur, "Efficacy of dexmedetomidine as an adjuvant in paravertebral block in breast cancer surgery," Journal of Anesthesia, vol. 30, no. 2, pp. 252-260, 2016.

[16] G. Fritsch, T. Danninger, K. Allerberger, A. Tsodikov, T. K. Felder, and M. Kapeller, "Dexmedetomidine added to ropivacaine extends the duration of interscalene brachial plexus blocks for elective shoulder surgery when compared with ropivacaine alone," Regional Anesthesia and Pain Medicine, vol. 39, no. 1, pp. 37-47, 2014.

[17] K. E. Boghdadly, C. Madjdpour, and K. J. Chin, "Thoracic paravertebral blocks in abdominal surgery - a systematic review of randomized controlled trials," British Journal of Anaesthesia, vol. 117, no. 3, pp. 297-308, 2016.

[18] F. W. Abdallah and R. Brull, "Facilitatory effects of perineural dexmedetomidine on neuraxial and peripheral nerve block: a systematic review and meta-analysis," British Journal of Anaesthesia, vol. 110, no. 6, pp. 915-925, 2013.

[19] X. Zeng, J. Jiang, L. Yang, and W. Ding, "Epidural dexmedetomidine reduces the requirement of propofol during total intravenous anaesthesia and improves analgesia after surgery in patients undergoing open thoracic surgery," Scientific Reports, vol. 7, no. 1, p. 3992, 2017.

[20] E. Koksal, D. Karakaya, B. Can et al., "Intracerebroventricular application of dexmedetomidine produces antinociception and does not cause neurotoxicity in rats," Balkan Medical Journal, vol. 30, no. 4, pp. 355-361, 2013.

[21] F. Celik, C. Göçmez, K. Kamaşak, A. Tufek, A. Guzel, and O. Tokgoz, "The comparison of neuroprotective effects of intrathecal dexmedetomidine and metilprednisolone in spinal cord injury," International Journal of Surgery, vol. 11, no. 5, pp. 414-418, 2013.

[22] V. Mahendru, A. Tewari, S. Katyal, A. Grewal, M. Singh, and R. Katyal, "A comparison of intrathecal dexmedetomidine, clonidine, and fentanyl as adjuvants to hyperbaric bupivacaine for lower limb surgery: a double blind controlled study," Journal of Anaesthesiology Clinical Pharmacology, vol. 29, no. 4, pp. 496-502, 2013.

[23] K. W. Tam, S. Y. Chen, T. W. Huang, C. C. Lin, C. M. Su, and C. L. Li, "Effect of wound infiltration with ropivacaine or bupivacaine analgesia in breast cancer surgery: a metaanalysis of randomized controlled trials," International Journal of Surgery, vol. 22, pp. 79-85, 2015.

[24] Y. Lin, B. He, J. Chen, and Z. Wang, "Can dexmedetomidine be a safe and efficacious sedative agent in post-cardiac surgery patients? a meta-analysis," Critical Care, vol. 16, no. 5, p. R169, 2012.

[25] B. Hong, C. Lim, H. Kang, H. Eom, Y. Kim, and H. J. Cho, "Thoracic paravertebral block with adjuvant dexmedetomidine in video-assisted thoracoscopic surgery: a randomized, double-blind study," Journal of Clinical Medicine, vol. 8, 2019. 